\title{
INFLUENCE OF CONVECTIVE DRYING TYPE ON PHENOLIC COMPOUND COMPONENTS OF THE EGYPTIAN HONEY BEE POLLENS
}

\author{
By \\ EMAD A. NAFEA ${ }^{1}$, EHAB W. ZIDAN ${ }^{1}$, DOAA S. FARGHALY ${ }^{2^{\star}}$ \\ AND ASMAA M. FAWZY',3
}

Department of Bee Research, Plant Protection Research Institute ${ }^{1}$, ARC, Dokki, Giza, and Faculty of Science ${ }^{2}$, Al -Azhar, University for Girls Branch, Egypt, and Department of Biology ${ }^{3}$, Faculty of Science, Taibah University,

Saudi Arabia (*Correspondence:dsaadfarghaly@yahoo.com)

\section{Abstract}

The present work determined theefficacy of three drying methods, $41^{\circ} \mathrm{C}$ sun drying (SD), $32^{\circ} \mathrm{C}$ shadow drying (ShD) and $32,40 \& 50^{\circ} \mathrm{C}$ incubator (ID) on the percentage of moisture loses and phenolic compounds content of pollen grains throughout $72 \mathrm{~h}$. SD \& ShD drying bee pollens showed the highest number of phenolic compound after $72 \mathrm{~h}$. with total peak of $11388228 \& 14374500$ respectively. The $32^{\circ} \mathrm{C}, 40^{\circ} \mathrm{C} \& 50^{\circ} \mathrm{C}$ ID of bee pollens showed the highest number of phenolic compounds after 24h., with total peak of 12429414,12078595 \& 12578030 respectively.

Key words: Honey bee, Pollens, Drying, Convective, Phenolic compounds.

\section{Introduction}

Bee pollen is a beehive product obtained by honey bees (Apis mellifera L.) gathering millions of floral pollen grains and mixing it with plant nectar and bee saliva rich in enzymes, thus transforming its composition and improving its therapeutically potential (Cocan et al, 2005). The bee pollen drying aimed to remove water to minimize microbial spoilage. There was a significant reduction in weight and volume contributing to reduce the cost of handling, storage and distribution (Sokhansanj and Jayas, 1995). Bee pollen is sold as fresh and dried. Fresh bee pollen needs to be refrigerated between $5^{\circ} \mathrm{C} \& 10^{\circ} \mathrm{C}$ to keep its quality. The drying preserves the bee pollen for longer time at room temperature without refrigeration by preventing rapid fermentation and microbial spoilage, providing ease of marketability for bee pollen and increasing profit of the beekeepers (Barajas et al, 2012). Sometimes, the sun drying of bee pollen is used inappropriate because of considerable process time, increased microbial spoilage during drying process and lower with sanitary conditions. The hot-air drying is a suitable and frequently used in a commercial product for reasonable process time, better sanitary and drying control conditions (Crapiste and Rotstein, 1997). Mostly the collected pollen pellets contain impurities, which should be removed, most efficiently by air specially constructed purifiers. The air should be free of dust and bacteria. In order to have minor loses of nutrients, pollen should be stored in a cool, dry place in well closed glass or plastic containers (Bogdanov, 2004). Nutrient content of pollen changes due to storage. Pollen is best dried in an electric oven, where humidity can continuously escape. The feasible temperature was $40^{\circ} \mathrm{C}$ and the drying time should be as short as possible to avoid loses of volatile compounds until the humidity was $6 \%$ or lower. Such pollen remains stable during storage for 15 months. Pollen containing more than $6 \%$ of water easily ferment upon storage. Storage for one year or more reduce the pollen free radical scavenging capacity (Campos et al, 2008). Bee pollen with high moisture content (20$30 \mathrm{~g}$ water $/ 100 \mathrm{~g}$ product in wet basis or 25 $42.9 \mathrm{~g}$ water $/ 100 \mathrm{~g}$ dry solid basis) perished after a short period of time from harvest as being highly susceptible to microbial attacks (De-Melo et al,2016). Low temperature may lead to formation of reactive oxygen species (ROS), as superoxide radicals, hydrogen peroxide, hydroxyl-radical and single oxygen. To reduce the oxidation damage, plants use enzymatic and non-enzymatic antioxidant mechanisms to scavenge ROS (Grene, 
2002). Among the chemical compounds in plants, secondary metabolites, in particular phenolic compounds (phenols and flavonoids) are of great importance in plant environment relationships (Rezanejad, 2009). These compounds are of a particular interest due to their involvement response of plant to environmental stress as low temperature (Robles et al, 2003). Natural antioxidants are found in pollen (LeBlank et al, 2009; De Arruda et al, 2013). Vitamins A, E, C, B-vitamins, niacin, rutin, polyphenols, and selenium compounds are widely found in pollen (Bonvehi, et al, 2001). Pollen grain contains bioactive compounds, which if present even in small amount, play an important role on human health. The bee pollen contains carotenoids, phenolic compounds and in particular flavonoids produced from plants' metabolism possessing numerous phenolic compounds with antioxidant activity (Bogdanov, 2006; Medeiros et al, 2008; Feás et al, 2012). The polyphenols are antioxidants with redox properties act as reducing agents, hydrogen donators, and singlet oxygen quenchers (Campone et al, 2014; Ranneh et al, 2018). Fanaliet al. (2013) found nine polyphenols: $o-, p$-coumaricacid, ferulic acid, myricetin, cinnamic acid, quercetin, naringenin, hesperitin and kaempferol. All analytics, with the exception of $p$-coumaric acid and myricetin partially co-eluted with other pollen components, were also quantified in sample. $\mathrm{Lv}$ et al. (2015) identified pollen samples from Qinghai-Tibetan Plateau by HPLC-DADAPCI/MS, quercetin and campperol, without routine and isoramnetin detection.

This work aimed to study the influence of convective type drying on phenolic compound components with optimum drying of bee pollens to produce high quality for this product.

\section{Materials and Methods}

The first hybrid of Carniolan bees race Apis mellifera L. of honey bee colonies were chosen in the apiary at Dokki, Giza 2018.
The pollen grains used in this experiment were collected by honeybees from multiflora were collected as corbicular pellets removed from the pollen baskets on the hind legs of bees as they passed through pollen traps attached to honeybee hives.

After the pollen grains carefully collection from pollen traps, they were exposed direct ly to different temperatures as the following: Sun rays dry (SD) $41^{\circ} \mathrm{C}$, Shadow dry (ShD) $32^{\circ} \mathrm{C}$ and incubator dry (ID) $32,40 \& 50^{\circ} \mathrm{C}$. All treated materials were collected after 24 , $48 \& 72 \mathrm{~h}$., and then the pollen grains were stored at $-20^{\circ} \mathrm{C}$ until analysis.

Moisture reduction $\%=($ weight pollen post temperature exposure/weight pollen before) - 1X 100.

Determination of the phenolic compounds: Preparing of $10 \%$ pollen solution, one $\mathrm{g}$ of pollen was solved in $10 \mathrm{ml}$ ethanol $70 \%$, and then kept in closed glass tubes for analysis by HPLC instrument. Identification of the phenolic compounds of bee pollen samples was done by a JASCO, using a hypersil C18 reversed- phase column $(250 \times 4.66 \mathrm{~mm})$ with $5 \mu \mathrm{m}$ particle size. All chemicals and solvents used were in HPLC spectral grade, as well as the standard phenolic compounds were purchased (Sigma).

Statistical analysis: Data were analyzed in (ANOVA) a randomized complete block design and means were compared $(\mathrm{p} \leq 0.05)$ by Duncan's multiple rang test. All analyses were performed by using a version 20 of the software SPSS.

\section{Results}

The $40^{\circ} \mathrm{C} \& 50^{\circ} \mathrm{C}$ ID had the highest significant differences values of the moisture losses (ML\%) of bee pollen compared with others after $24 \mathrm{~h}$., but with neither significant variation between all temperatures on moisture reduction at $48 \& 72 \mathrm{~h}$. nor temperatures using the three times $(24,48 \& 72 \mathrm{~h}$.) except for $41^{\circ} \mathrm{C} \mathrm{SD}$, which showed significantly the highest value $2.32 \mathrm{ML} \%$ after 48h (Tab. 1). 
Table 1: Effect of different treatments and temperature degree on reduction of moisture percentage of bee pollens.

\begin{tabular}{|c|c|c|c|c|c|c|c|c|c|c|c|c|c|c|c|c|c|c|}
\hline \multirow{2}{*}{$\begin{array}{l}\text { Treatment } \\
\text { Time }\end{array}$} & \multicolumn{3}{|c|}{$41^{\circ} \mathrm{C} \mathrm{SD}$} & \multicolumn{3}{|c|}{$32^{\circ} \mathrm{CShD}$} & \multicolumn{3}{|c|}{$32^{\circ} \mathrm{C}$ ID } & \multicolumn{3}{|c|}{$40^{\circ} \mathrm{CID}$} & \multicolumn{3}{|c|}{$50^{\circ} \mathrm{CID}$} & \multirow[t]{2}{*}{$\mathrm{F}$} & \multirow{2}{*}{$\mathrm{LSD}_{0.05}$} & \multirow{2}{*}{$\begin{array}{l}\mathrm{P} \leq \\
0.05\end{array}$} \\
\hline & W B & W A & $\% \mathrm{M}$ & W B & W A & $\% \mathrm{M}$ & W B & W A & $\% \mathrm{M}$ & W B & W A & $\% \mathrm{M}$ & W B & W A & $\% \mathrm{M}$ & & & \\
\hline \multirow[t]{3}{*}{$24 \mathrm{~h}}$. & 42.70 & 42.13 & 1.35 & \begin{tabular}{|l|}
49.72 \\
\end{tabular} & 49.44 & 0.57 & 43.82 & 43.66 & 0.37 & 41.47 & 40.86 & 1.49 & 52.29 & 51.62 & 1.30 & \multirow{4}{*}{$\begin{array}{l}26.8 \\
9\end{array}$} & \multirow[t]{4}{*}{1.15} & \multirow{4}{*}{$\begin{array}{l}0.000 \\
* * *\end{array}$} \\
\hline & 42.24 & 41.75 & 1.20 & \begin{tabular}{|l|}
50.16 \\
\end{tabular} & 49.81 & 0.70 & 40.48 & 40.31 & 0.42 & 53.60 & \begin{tabular}{|l|}
52.70 \\
\end{tabular} & 1.71 & 55.77 & 54.77 & 1.82 & & & \\
\hline & 50.38 & 49.96 & 0.84 & \begin{tabular}{|l|}
49.50 \\
\end{tabular} & 49.19 & 0.63 & 41.07 & 40.98 & 0.22 & 56.84 & \begin{tabular}{|l|}
55.69 \\
\end{tabular} & 2.07 & 57.09 & 56.15 & 1.67 & & & \\
\hline Mean & 45.11 & 44.61 & $1.13^{\mathrm{Bb}}$ & \begin{tabular}{|l|}
49.79 \\
\end{tabular} & 49.48 & $0.63^{\mathrm{Ac}}$ & 41.79 & 41.65 & $0.34^{\mathrm{Ac}}$ & 50.64 & \begin{tabular}{|l|}
49.84 \\
\end{tabular} & $1.77^{\mathrm{ABa}}$ & 55.05 & 54.18 & $1.60^{\mathrm{Aa}}$ & & & \\
\hline \multirow[t]{3}{*}{$48 \mathrm{~h}}$. & 43.45 & 42.50 & 2.24 & \begin{tabular}{|l|}
51.44 \\
\end{tabular} & 50.79 & 1.28 & 38.53 & 38.15 & 1.00 & 55.54 & 55.11 & 0.78 & 40.69 & 40.24 & 1.12 & \multirow[t]{4}{*}{2.57} & \multirow[t]{4}{*}{2.99} & \multirow[t]{4}{*}{$0.103^{\mathrm{ns}}$} \\
\hline & 43.76 & 42.81 & 2.22 & \begin{tabular}{|l|}
55.16 \\
\end{tabular} & \begin{tabular}{|l|}
54.58 \\
\end{tabular} & 1.07 & 53.81 & \begin{tabular}{|l|}
53.61 \\
\end{tabular} & 0.37 & 53.05 & \begin{tabular}{|l|}
52.73 \\
\end{tabular} & 0.61 & 41.10 & 40.62 & 1.18 & & & \\
\hline & 41.15 & 40.16 & 2.47 & 51.19 & 51.05 & 0.28 & 39.95 & \begin{tabular}{|l|}
38.81 \\
\end{tabular} & 2.93 & 46.91 & 46.59 & 0.69 & 38.39 & 37.89 & 1.32 & & & \\
\hline Mean & 42.79 & 41.82 & $2.32^{\mathrm{Aa}}$ & 52.60 & 52.14 & $0.88^{\mathrm{Ab}}$ & 44.10 & 43.52 & $1.43^{\mathrm{Aab}}$ & 51.83 & 51.48 & $0.69^{\mathrm{Bb}}$ & 40.06 & 39.58 & $1.21^{\mathrm{Bab}}$ & & & \\
\hline \multirow[t]{3}{*}{$72 \mathrm{~h}}$. & 50.90 & \begin{tabular}{|l|}
50.19 \\
\end{tabular} & 1.41 & \begin{tabular}{|l|}
41.12 \\
\end{tabular} & 40.59 & 1.31 & 50.19 & \begin{tabular}{|l|}
49.66 \\
\end{tabular} & 1.07 & 41.78 & 41.28 & 1.21 & 35.63 & 35.13 & 1.43 & \multirow[t]{4}{*}{1.11} & \multirow[t]{4}{*}{1.143} & \multirow[t]{4}{*}{$0.41^{\mathrm{ns}}$} \\
\hline & 48.35 & \begin{tabular}{|l|}
47.71 \\
\end{tabular} & 1.34 & 41.27 & 40.75 & 1.28 & 38.88 & \begin{tabular}{|l|}
38.43 \\
\end{tabular} & 1.16 & 41.72 & 41.31 & 0.99 & 41.21 & 40.62 & 1.46 & & & \\
\hline & 48.80 & 48.21 & 1.22 & \begin{tabular}{|l|}
49.45 \\
\end{tabular} & 49.03 & 0.86 & 43.66 & 42.91 & 1.74 & 56.84 & 55.99 & 1.52 & 49.82 & 49.03 & 1.61 & & & \\
\hline Mean & 49.35 & 48.70 & $1.32^{\mathrm{Ba}}$ & \begin{tabular}{|l|}
43.96 \\
\end{tabular} & 43.46 & $1.15^{\mathrm{Aa}}$ & 44.24 & 43.67 & $1.31^{\mathrm{Aa}}$ & 46.78 & 45.86 & $1.24 \mathrm{~A} \mathrm{a}$ & 42.22 & 41.59 & $1.50^{\mathrm{ABa}}$ & & & \\
\hline Total & \multicolumn{3}{|c|}{4.77} & \multicolumn{3}{|c|}{2.66} & \multicolumn{3}{|c|}{3.08} & \multicolumn{3}{|c|}{3.70} & \multicolumn{3}{|c|}{4.31} & & & \\
\hline $\mathrm{F}$ & \multicolumn{3}{|c|}{25.14} & \multicolumn{3}{|c|}{2.28} & \multicolumn{3}{|c|}{2.88} & \multicolumn{3}{|c|}{4.43} & \multicolumn{3}{|c|}{4.56} & & & \\
\hline $\operatorname{LSD}_{0.05}$ & & .0 .99 & & & 2.31 & & & 3.81 & & & 2.90 & & & 0.82 & & & & \\
\hline $\mathrm{P} \leq 0.05$ & & $0.0012 *$ & & & $0.1837^{\mathrm{n}}$ & & & $0.1329^{11}$ & & & 0.0657 & & & 0.0624 & & & & \\
\hline
\end{tabular}

Means data with different capital letters in column and small letters in row at $\mathrm{p} \leq 0.05, \mathrm{WB}=\mathrm{Bee}$ pollen weight before treatment, $\mathrm{WA}=\mathrm{Bee}$ pollen weight after treatment, $\% \mathrm{M}=$ moisture percentage, $\mathrm{SD}=$ Sun drying, $\mathrm{ShD}=$ Shadow drying, $\mathrm{ID}=\mathrm{Incubator}$ drying.

Table 2: Influence of convective drying by different methods on phenolic compounds component in bee pollens $(\mathrm{mg} / 100 \mathrm{~g})$

\begin{tabular}{|c|c|c|c|c|c|c|c|}
\hline \multirow{2}{*}{ Standards } & \multirow{2}{*}{ Control } & \multicolumn{3}{|c|}{ Sun rays $\left(41^{\circ} \mathrm{C}\right)$} & \multicolumn{3}{|c|}{$\operatorname{Shadow}\left(32^{\circ} \mathrm{C}\right)$} \\
\hline & & $24 \mathrm{~h}$ & $48 \mathrm{~h}$ & $72 \mathrm{~h}$ & $24 \mathrm{~h}$ & $48 \mathrm{~h}$ & $72 \mathrm{~h}$ \\
\hline Pyrogallic acid: *benzene-1,2,3-triol & 0.0000 & 0.0000 & 0.0000 & 0.9731 & 0.0000 & 0.0000 & 0.0000 \\
\hline *Phenol & 0.0115 & 0.0000 & 0.0143 & 0.0000 & 0.0011 & 0.0000 & 0.0178 \\
\hline Salicylic acid : 2 -hydroxybenzoic acid & 0.0028 & 0.3019 & 0.0000 & 0.0000 & 0.0000 & 0.0000 & 0.0035 \\
\hline Ferulic acid: *3-(4-hydroxy-3-methoxy-phenyl)prop-2- enoic acid & 0.0900 & 0.0000 & 0.0000 & 0.0407 & 0.0562 & 0.0323 & 0.0011 \\
\hline *3,5-dimethoxyphenol & 0.0000 & 0.1350 & 0.0347 & 0.0000 & 0.0000 & 0.0000 & 0.1095 \\
\hline p-Coumaric acid: *3-(4-hydroxyphenyl)prop-2-enoic acid & 0.0004 & 0.0000 & 0.0000 & 0.0000 & 0.0257 & 0.0120 & 0.0045 \\
\hline Phenolphthalein: *3,3-Bis(4-hydroxyphenyl)-2-benzofuran-1(3H)-one & 0.0000 & 0.0000 & 0.0000 & 0.0000 & 0.0378 & 0.1756 & 0.0020 \\
\hline Eugenol:*4-Allyl-2-methoxyphenol & 0.0000 & 0.0000 & 0.0000 & 0.0000 & 0.0000 & 0.0000 & 0.1853 \\
\hline Cinnamic acid: * (E)-3-phenylprop-2-enoic acid & 0.0000 & 0.0051 & 0.0008 & 0.0010 & 0.0000 & 0.0000 & 0.0000 \\
\hline Gallic acid: *3,4,5-Trihydroxy benzoic acid & 0.0000 & 0.0000 & 0.0000 & 0.0000 & 0.0000 & 0.0000 & 0.0000 \\
\hline Resorcinol: *benzene-1,3-diol & 0.0000 & 0.0000 & 0.5218 & 0.7305 & 0.0000 & 0.0000 & 0.0000 \\
\hline Protocetchol & 0.0000 & 0.0000 & 0.0000 & 0.0000 & 0.0000 & 0.0000 & 0.0000 \\
\hline$p$-OH benzoic acid $* 4$-Hydroxybenzoic acid & 0.0011 & 0.0000 & 0.0012 & 0.0001 & 0.0000 & 0.0000 & 0.0000 \\
\hline Caffeic acid: *3-(3,4-dihydroxyphenyl)prop-2-enoic acid & 0.0000 & 0.0029 & 0.0000 & 0.0000 & 0.0000 & 0.0000 & 0.0000 \\
\hline Vanillin: *4-hydroxy-3-methoxy-benzaldehyde & 0.0000 & 0.0000 & 0.0058 & 0.0009 & 0.0000 & 0.0000 & 0.0000 \\
\hline Quercetin: *2-(3,4-dihydroxyphenyl)-3,5,7-trihydroxy-chromen-4-one & 0.0037 & 0.0141 & 0.0026 & 0.0151 & 0.0000 & 0.0040 & 0.0000 \\
\hline Pinocembrin: *(2S)-5,7-dihydroxy-2-phenyl-2,3 dihydrochromen-4-one & 0.0000 & 0.0235 & 0.0000 & 0.0064 & 0.0047 & 0.0000 & 0.0000 \\
\hline Chrysin: *5,7-dihydroxy-2-phenyl-chromen-4-one ${ }_{4}$ & 0.0000 & 0.0000 & 0.0000 & 0.0000 & 0.0000 & 0.0000 & 0.0000 \\
\hline Galangin: *3,5,7-trihydroxy-2-phenyl-chromen-4-one & 0.0000 & 0.0000 & 0.0000 & 0.0000 & 0.0000 & 0.0000 & 0.0000 \\
\hline Acacetin: *5,7-dihydroxy-2-(4- methoxyphenyl)chromen-4-one & 0.0000 & 0.0000 & 0.0000 & 0.0000 & 0.0000 & 0.0000 & 0.0000 \\
\hline 3,5 dihydroxyisoflavone: *3.5-Dihydroxy-3-(4-hydroxyphenyl)chromen-4-1 & 0.0000 & 0.0000 & 0.0000 & 0.0002 & 0.0000 & 0.0000 & 0.0000 \\
\hline Pinostrobin: *5,7-dihydroxy-2-phenyl-chroman-4-one & 0.0000 & 0.0000 & 0.0000 & 0.0000 & 0.0000 & 0.0000 & 0.0000 \\
\hline Total Peak Area & 13139199 & 10644580 & 12148065 & 11388228 & 10730331 & 12112796 & 14374500 \\
\hline
\end{tabular}

The influence of convective drying by the different methods on phenolic compounds value in bee pollens: In $41^{\circ} \mathrm{C}$ SD phenolic content after $24 \mathrm{~h}$., were seven compounds: caffeic acid, salicylic acid, 3, 5-dimethoxyphenol, cinnamic acid, quercetin, pinocembrin and 3, 5-dihydroxy isoflavon. Also, seven phenolic compounds were found after 48h.: Resorcinol, p-ohbenzoic, phenol, vanillin, 3, 5-dimethoxyphenol, cinnamic acid, quercetin, but increased to nine phenolic compounds. After $72 \mathrm{~h}$ the resulting phenolic compounds kinds and percentage were not stability. The more stable phenolic compounds were cinnamic acid and quercetin. In $32^{\circ} \mathrm{C} \mathrm{ShD} \mathrm{the} \mathrm{number} \mathrm{of} \mathrm{phenolic} \mathrm{comp-}$ ound unstable through $72 \mathrm{~h}$. were five, four $\&$ seven respectively, after the first $24 \mathrm{~h}$. ferulic acid was the highest compound 0.0562 $\mathrm{mg} / 100 \mathrm{~g}$, while phenol phthaleinit $(0.1756$ $\mathrm{mg} / 100 \mathrm{~g}$ ) was the highest one after $48 \mathrm{~h}$. (Table 2 continued): 


\begin{tabular}{|c|c|c|c|c|c|c|c|c|c|c|}
\hline \multirow{2}{*}{ Standards } & \multirow{2}{*}{ Control } & \multicolumn{3}{|c|}{ Incubator $\left(32^{\circ} \mathrm{C}\right)$} & \multicolumn{3}{|c|}{ Incubator $\left(40^{\circ} \mathrm{C}\right)$} & \multicolumn{3}{|c|}{ Incubator $\left(50^{\circ} \mathrm{C}\right)$} \\
\hline & & $24 \mathrm{~h}$ & $48 \mathrm{~h}$ & $72 \mathrm{~h}$ & $24 \mathrm{~h}$ & $48 \mathrm{~h}$ & $72 \mathrm{~h}$ & $24 \mathrm{~h}$ & $48 \mathrm{~h}$ & $72 \mathrm{~h}$ \\
\hline Pyrogallic acid: *benzene-1,2,3-triol & 0.0000 & 1.2697 & 0.0000 & 0.0000 & 1.3219 & 1.2079 & 0.0000 & 0.7806 & 0.4341 & 0.7922 \\
\hline *phenol & 0.0115 & 0.0000 & 0.0000 & 0.0122 & 0.0000 & 0.0000 & 0.0000 & 0.0000 & 0.0000 & 5.5771 \\
\hline Salicylic acid: *2-hydroxybenzoic acid & 0.0028 & 2.1413 & 0.0000 & 2.5651 & 0.0020 & 0.0000 & 0.9681 & 0.0036 & 0.1026 & 0.0000 \\
\hline $\begin{array}{l}\text { Ferulic acid: *3-(4-hy droxy-3-methoxy-phenyl)prop-2- } \\
\text { enoic acid }\end{array}$ & 0.0900 & 0.0169 & 0.0000 & 0.0000 & 0.0036 & 0.0000 & 0.0182 & 0.0963 & 0.0000 & 0.2997 \\
\hline *3,5-dimethoxyphenol & 0.0000 & 0.0000 & 0.5807 & 0.1258 & 0.0000 & 0.0000 & 0.0000 & 0.0000 & 0.0000 & 1.2907 \\
\hline $\begin{array}{l}p \text {-Coumaric acid: *3-(4-hydroxyphenyl)prop-2-enoic } \\
\text { acid }\end{array}$ & 0.0004 & 0.1547 & 0.0000 & 0.0000 & 0.0022 & 0.07725 & 0.0000 & 0.0000 & 2.9091 & 0.0000 \\
\hline $\begin{array}{l}\text { Phenolphthalein: *3,3-Bis(4-hydroxyphenyl)-2- } \\
\text { benzofuran-1 }(3 \mathrm{H}) \text {-one }\end{array}$ & 0.0000 & 0.0000 & 0.0000 & 0.0000 & 0.0000 & 0.0000 & 0.0000 & 0.0000 & 0.0000 & 0.0000 \\
\hline Eugenol:*4-Allyl-2-methoxyphenol & 0.0000 & 0.0000 & 0.0000 & 0.0000 & 0.0000 & 0.0000 & 0.0000 & 0.0000 & 0.0000 & 0.0000 \\
\hline Cinnamic acid: * $(E)$-3-phenylprop-2-enoic acid & 0.0000 & 0.0009 & 0.0000 & 0.0000 & 0.0014 & 0.0285 & 1.8323 & 0.0000 & 7.7647 & 0.0000 \\
\hline Gallic acid: $* 3,4,5$-Trihydroxy benzoic acid & 0.0000 & 0.0000 & 0.0129 & 0.0000 & 0.0000 & 0.0000 & 0.0000 & 0.0000 & 0.0000 & 0.0000 \\
\hline Resorcinol: *benzene-1,3-diol & 0.0000 & 0.3364 & 0.0000 & 0.0000 & 0.2900 & 0.0000 & 0.0000 & 0.8725 & 0.0000 & 0.0000 \\
\hline Protocetchol & 0.0000 & 0.0051 & 0.0000 & 0.0000 & 0.0044 & 0.0000 & 0.0000 & 0.0060 & 0.0000 & 0.0000 \\
\hline p-OH benzoic acid *4-Hydroxybenzoic acid & 0.0011 & 0.0011 & 0.0000 & 1.0516 & 0.0011 & 0.0000 & 1.1897 & 0.0009 & 7.9380 & 9.8671 \\
\hline Caffeic a cid: *3-(3,4-dihy droxypherryl)prop-2-enoic a cid & 0.0000 & 0.0000 & 0.0000 & 0.0000 & 0.0000 & 1.4652 & 0.0000 & 0.0000 & 0.0000 & 0.0000 \\
\hline Vanillin: *4-hydroxy-3-methoxy-benzaldehyde & 0.0000 & 0.0000 & 0.0000 & 0.0000 & 0.0000 & 0.0000 & 0.0000 & 0.0000 & 6.0731 & 0.0000 \\
\hline $\begin{array}{l}\text { Quercetin: *2-(3,4-dihy droxyphenyl)-3,5,7-trihy droxy- } \\
\text { chromen-4-one }\end{array}$ & 0.0037 & 0.0234 & 0.0000 & 4.2376 & 0.0218 & 0.0000 & 1.0311 & 0.0247 & 0.0163 & 4.0814 \\
\hline $\begin{array}{l}\text { Pinocembrin: *(2S)-5,7-dihy droxy-2-phenyl-2,3 } \\
\text { dihy drochromen-4-one }\end{array}$ & 0.0000 & 0.0000 & 0.0000 & 0.0000 & 0.0084 & 8.9172 & 0.0000 & 0.0000 & 7.3439 & 0.0000 \\
\hline Chrysin: $* 5,7$-dihydroxy-2-phenyl-chromen-4-one 4 & 0.0000 & 0.0000 & 0.0000 & 0.0000 & 0.0000 & 0.0000 & 1.8292 & 0.0000 & 0.0000 & 0.0000 \\
\hline Galangin: *3,5,7-trihy droxy-2-phenyl-chromen-4-one & 0.0000 & 0.0000 & 0.0000 & 0.0000 & 0.0000 & 0.0000 & 1.7936 & 0.0000 & 0.0000 & 0.0000 \\
\hline $\begin{array}{l}\text { Acacetin: } * 5,7 \text {-dihydroxy-2-(4- } \\
\text { methoxyphenyl)chromen-4-one }\end{array}$ & 0.0000 & 0.0000 & 0.0000 & 0.0000 & 0.0000 & 0.0000 & 5.0083 & 0.0000 & 0.0000 & 0.0000 \\
\hline $\begin{array}{l}\text { 3,5 dihydroxyisoflavone: *3.5-Dihy droxy-3-(4- } \\
\text { hydroxyphenyl)chromen-4-one }\end{array}$ & 0.0000 & 0.0002 & 0.0000 & 0.0000 & 0.0000 & 0.0000 & 0.0000 & 0.0000 & 0.0000 & 0.0000 \\
\hline Pinostrobin: * 5,7-dihy droxy-2-phenyl-chroman-4-one & 0.0000 & 0.0000 & 0.0000 & 0.0000 & 0.0000 & 0.0000 & 0.0000 & 0.0000 & 0.0000 & 0.0000 \\
\hline Total Peak Area & 13139199 & 12429414 & 10377088 & 11482122 & 12078595 & 11204660 & 11505611 & 12578030 & 10515447 & 12307766 \\
\hline
\end{tabular}

Eugenol $(0.185 \mathrm{mg} / 100 \mathrm{~g})$ was the highest compound after $72 \mathrm{~h}$., while the more stable compounds were ferulic acid, coumaric acid and phenolphthalein. In $32^{\circ} \mathrm{C}$ ID phenolic compounds were 10 after the first $24 \mathrm{~h}$., and decreased to two \& five after $48 \& 72$ h., respectively, without stable phenolic ones. In $40^{\circ} \mathrm{C}$ ID ten phenolic compounds were detected after the first $24 \mathrm{~h}$., decreased to five after $48 \mathrm{~h}$., and increased to eight after $72 \mathrm{~h}$. The cinnamic acid was stable after $72 \mathrm{~h}$. In $50^{\circ} \mathrm{C}$ ID seven compounds were found after $24 \mathrm{~h}$., increased to eight and decreased to six compounds after 48 \& 72h., respectively, with three phenolic ones were stable in this treatment pyrogallic acid, p-oh benzoic and quercetin. Stable phenolic compounds at $41{ }^{\circ} \mathrm{C} \mathrm{SD}$ were cinnamic acid and quercetin. Quercetin increased after $72 \mathrm{~h}$., to $0.0151 \mathrm{mg} /$ $100 \mathrm{~g}$ compared to control $0.0037 \mathrm{mg} / 100 \mathrm{~g}$. Pinocembrin at $40^{\circ} \mathrm{C}$ ID after $48 \mathrm{~h}$ drying showed the highest $8.9172 \mathrm{mg} / 100 \mathrm{~g}$. It has the primary multifunctional flavonoid used in pharmaceutical industry. After $72 \mathrm{~h}$ drying ferulic acid was detected. Pyrogallic acid recorded $1.2697 \mathrm{mg} / 100 \mathrm{~g} \& 1.3219 \mathrm{mg} / / 100 \mathrm{~g}$ at $32^{\circ} \mathrm{C}$ ID $\& 40^{\circ} \mathrm{C}$ ID after $24 \mathrm{~h}$. respecti- vely, but with lowest amount was $0.434 \mathrm{mg} /$ $100 \mathrm{~g} \& 0.7806 \mathrm{mg} / 100 \mathrm{~g}$ at $50^{\circ} \mathrm{C}$ ID after $48 \mathrm{~h}$ $\& 24 \mathrm{~h}$., respectively. Most of the phenolic compounds useful properties increased at $40^{\circ} \mathrm{C}$ ID \& $50^{\circ} \mathrm{C}$ ID. p-oh benzoic for instance reached $1.1897 \mathrm{mg} / 100 \mathrm{~g} \& 9.8671 \mathrm{mg} /$ $100 \mathrm{~g}$ for $40^{\circ} \mathrm{C}$ ID and $50^{\circ} \mathrm{C}$ ID, respectively. At $40^{\circ} \mathrm{C}$ ID vanillin recorded $6.0731 \mathrm{mg} / 100$ $\mathrm{g}$ as compared to $41^{\circ} \mathrm{C} \mathrm{SD}$, which recorded $0.0058 \mathrm{mg} / 100 \mathrm{~g}$.

\section{Discussion}

Temperature is one of the most critical factors that affect product quality and shelflife. In general, in literature, the effects of heating on the degradation of biologically active compounds have been assessed in terms of the levels of phenolic compounds (Georgé et al, 2011). Barajas et al. (2012) found that the drying process of bee pollens at $45^{\circ} \mathrm{C}$ had the moisture content $(7-8 \%)$, but at $35^{\circ} \mathrm{C}$ moisture content increased to reach $9-11 \%$. Dried bee pollens at $35^{\circ} \mathrm{C} \& 45^{\circ} \mathrm{C}$ were not enough to determine parameters in drying kinetics. Hot air drying affected organoleptic properties, \& pollens. Midilli et al. (2000) dried bee pollens at $45^{\circ} \mathrm{C}$ in solar and electric assisted dryer and measured weight 
changes of pollens during the drying. They were unable to study drying kinetics of bee pollen at one temperature. Many factors contribute to the composition of bee pollen, as the plant source, climatic conditions, soil characters and the beekeeper activities (Morais et al, 2011; Estevinho et al, 2012). The relative instability of most phenolic compounds from plants showed their sensitivities to drying method (Lim and Murtijaya, 2007). Phenolic constituents concentration depends on various factors, including plant species used by the bees, the plant health, season, environmental factors...etc. (Kücük et al, 2007). The commonest phenolic acids were chlorogenic, gallic, ferulic, cinnamic (Almaraz-Abarca et al, 2004), caffeic acids (Kędzia, 2008) and hydroxyl cinnamic, ortho-coumaric and paracoumaric acids (Bonvehi et al, 2001; Almaraz-Abarca et al, 2007). Šarić et al. (2009) in phenolic composition of Croatian bee pollens by HPLC analysis detected seven compounds, which were flavonol (pinocembrin), flavanols (quercetin, kaempferol, galangin, \& isorhamnetin), flavones (chrysin) and phenylpropanoids (caffeic acid).

In Egyptian bee pollen, quercetin, rutin, catechin, epicatechin, kaempferol, apigenin, naringenin, and luteolin were detected (Mohdaly et al, 2015) that agreed with Buchner et al. (2006) who found that the thermal treatment of quercetin was the most important flavonoid widely used in human drugs. It has antimicrobial, anti-inflammatory, antioxidant and anticancer activities (Rasul et al, 2013). Ewald et al. (1999) found that some phenolic compounds, such as ferulic acid and $p$-coumaric acid, existed as free acids, soluble esters and insoluble esters in rice, corn and other pollen grains. Even when free phenolic acids are in low concentration in foods, they may increase in processed food underwent freezing or fermentation, and could affect by food processing as well, especially those involved in thermal treatment. The pyrogallic was used as antioxidant and as a topical anti-psoriatic (Budavari, 1996), also used in con-junction with ultraviolet B for resistant psoriasis (Siage, 1976). p-hydroxy benzoic acid, chemical obtained naturally and synthetically has anti-microbial, anti-algal, anti-mutagenic, anti-estrogenic, hypoglycemic, anti-in-flammatory, anti-platelet aggregating, nematicidal, antiviral, antioxidant...etc. (Manuja et al, 2013). Vanillin sensitive to sunlight, on heating gives $\mathrm{CO} \&$ $\mathrm{CO}_{2}$ with anti-microbial activity (Kumar et al, 2012). Isik et al. (2019) found that sun drying was the widely used traditional method for pollen drying, but with some disadvantages such as long drying times, microbial contamination risk, insect infestation susceptibility, large drying area, and high man power costs without control of drying conditions. Hot air drying has shorter time, low microbial contamina- tion risk, more effective sanitary conditions and better control of drying conditions. So hot air dried at $40^{\circ} \mathrm{C}$ was recommended for the drying of bee pollens.

\section{Conclusion}

Determination of the effect of drying temperature and drying time on Phenolic compounds, of bee pollen is important to obtain bee pollen products with high quality characteristics. Sun and shadow drying bee pollen showed highest phenolic compounds after $72 \mathrm{~h}$., with a peak area 11388228 \& 14374500 respectively. At $32^{\circ} \mathrm{C}, 40^{\circ} \mathrm{C} \&$ $50^{\circ} \mathrm{C}$ incubator drying of bee pollen showed highest compounds after $24 \mathrm{~h}$. with a peak area $12429414,12078595 \& 12578030$ respectively. Moisture reduction was of no significant in all drying methods after 48 \& $72 \mathrm{~h}$. Recommended for of bee pollens, drying was hot air at $32^{\circ} \mathrm{C} \mathrm{ShD,} \mathrm{32,} 40 \& 50^{\circ} \mathrm{C}$ incubator $24 \mathrm{~h}$., while $41^{\circ} \mathrm{C}$ SD $48 \mathrm{~h}$.

\section{References}

Almaraz-Abarca, N, da Graça Campos, M, Ávila-Reyes, JA, Jiménez, N, Carrol, HJ, Valdez, LS, 2004: Variability of antioxidant activity among honeybee-collected pollen of different botanical origin. Interciencia Caracas 29:574-8.

Almaraz-Abarca, N, da Graça Campos, M, Ávila-Reyes, JA, Naranjo-Jiménez, N, Carrol, HJ, et al, 2007: Antioxidant activity of polyphenolic extract of monofloral honeybee-collected 
pollen from mesquite (Prosopisjuliflora, Leguminosae). J. Food Compos. Anal. 20:119-24.

Barajas, J, Cortes-Rodriguez, M, RodríguezSandoval, E, 2012:Effect of temperature on the drying process of bee pollen from two zones of Colombia. J. Food Process Engin. 35, 1:134-48.

Bogdanov, S, 2004: Quality and standards of pollen and beeswax. Apiacta 38:334-41.

Bogdanov, S, 2006: Contaminants of bee products. Apidologie 37:1-18.

Bonvehí, JS, Torrentó, MS, Lorente, EC, 2001: Evaluation of polyphenolic and flavonoid compounds in honeybee-collected pollen produced in Spain. J. Agric. Food Chem. 49:1848-53.

Buchner, N, Krumbein, A, Rohn, S Kroh, L W, 2006: Effect of thermal processing on the flavonols rutin and quercetin. Rapid Commun. Mass Spectrom. 20:3229-35.

Budavari, S, 1996: The Merck Index, $12^{\text {th }}$ Edition, Whitehouse Station, NJ, Merck \& Co.

Campone, L, Piccinelli, AL, Pagano, I, Carabetta, S, Di Sanzo, R, et al,2014: Determination of phenolic compounds in honey using dispersive liquid-liquid microextraction. J. Chromatogr. A, 1334:9-15.

Campos, MGR, Bogdanov, S, Almeida-Muradian, LB, Szczesna, T, Mancebo, Y, et al, 2008: Pollen composition and standardization of analytical methods. J. Apic. Res. Bee World 47, 2:156-63.

Cocan, O, Mărghias, LA, Dezmirean, D, Laslo, L, 2005: Composition and biological activities of bee pollen: Review. USAMV-CN Bull. 61: 221-6.

Crapiste, G, Rotstein, E, 1997: Design and performance evaluation of dryers. In: Handbook of Food Engineering Practice.

De Arruda, VAS, Pereira, AAS, Freitas, AS, Barth, OM, Muradian, LB, 2013: Dried bee pollen: B complex vitamins, physicochemical and botanical composition. J. Food Compos. Anal. 29:100-5.

De-Melo, AAM, Estevinho, MLMF, Sattler, J AG, Souza, BR, Freitas, AS, et al, 2016: Effect of processing conditions on characteristics of dehydrated bee-pollen and correlation between quality parameters. Leben. Wissenschaft Tech. 65: 808-15.

Estevinho, LM, Rodrigues, S, Pereira, AP, Feás, X, 2012: Portuguese bee pollen: Palynological study, nutritional and microbiological evaluation. Inter. J. Food Sci. Tech. 47:429-35.
Ewald, C, Fjelkner-Modig, S, Johansson, K, Sjoholm, I, Akesson, B, 1999: Effect of processing on major flavonoids in processed onions, green beans, and peas. Food Chem. 64:231-5.

Fanali, C, Dugo, L, Rocco, A, 2013: Nano-liquid chromatography in nutritional analysis: Determination of polyphenols in bee pollen. J. Chrom. A. 1313:270-4.

Feás, X, Vázquez-Tato, MP, Estevinho, L, Seijas, JA, Iglesias, A, 2012: Organic bee pollen: Botanical origin, nutritional value, bioactive compounds: Antioxidant activity and microbiological quality. Molecules 17:8359-77.

Georgé, S, Tourniaire, F, Gautier, H, Goupy, P, Rock, E, Caris-Veyrat, C, 2011: Changes in the contents of carotenoids, phenolic compounds and vitamin $\mathrm{C}$ during technical processing and lyophilisation of red and yellow tomatoes. Food Chem. 124:1603-11.

Grene, R, 2002: Oxidative stress and acclimation mechanisms in plants. In: Somerville, CR, Myerowitz, EM (Eds.): The Arabidopsis Book. Am. Soc. Plant Biol., Rockville, MD, USA.

Isik, A, Ozdemir, M, Doymaz, I, 2019: Effect of hot air drying on quality characteristics and physicochemical properties of bee pollen. Food Sci. Tech. Campinas 39, 1:224-31.

Kędzia, B, 2008: Chemical composition and adaptogenic activity of honeybee-collected pollen. Part-1: Chemical composition. Postep. Fitoter. 1:47-58.

Kücük, M, Kolayh. S, Karaoglu, S, 2007: Biological activities and chemical composition of three honeys of different types from Anatolia. Food Chem. 100:526-34.

Kumar, R, Sharma, PK, Mishra, PSh, 2012: A review on the vanillin derivatives showing various biological activities. Inter. J. Pharm. Tech. Res. 4, 1:266-79.

LeBlanc, BW, Davis, OK, Boue, S, DeLucca, A, Deeby, T, 2009: Antioxidant activity of Sonoran Desert bee pollen. Food Chem.115:1299305.

Lim, YY, Murtijaya, J, 2007: Antioxidant properties of Phyllanthusamarusextracts as affected by different drying methods. LWT-Food Sci. Tech. 40:1664-9.

Lv, H, Wang, X, He, Y, 2015: Identification and quantification of flavonoid aglycones in rape bee 4 pollen from Qinghai-Tibetan Plateau by HPLC-DAD/APCI/MS. J. Food Comp. Anal. 38 :49-54. Sigma 
Manuja, R, Sachdeva, S, Jain, A, Chaudhary, J, 2013: A comprehensive review on biological activities of Phydroxy benzoic acid and its derivatives. Int. J. Pharm. Sci. Rev. Res. 22, 2:10915.

Medeiros, KCP, Figueiredo, CAV, Figueredo, TB, Freire, KRL, Santos, FAR, et al, 2008: Anti-allergic effect of bee pollen phenolic extract and myricetin in ovalbumin-sensitized mice. J. Ethno-pharm. 119:41-6.

Midilli, A, Olgun, H, Rzayev, P, Ayhan, T, 2000: Drying and conservation conditions of pollen. J. Sci. Food Agric. 80, 13:1973-80.

Mohdaly, AAA, Mahmoud, AA, Roby, MHH, Smetanska, I, Ramadan, MF, 2015: Phenolicextract from propolis and bee pollen: Composition, antioxidant and antibacterial activities. J. Food Bio. 39:538-47.

Morais, M, Moreira, L, Feás, X, Estevinho, L M, 2011: Honeybee-collected pollen from five Portuguese Natural Parks: Palynological origin, phenolic content, antioxidant properties and antimicrobial activity. Food Chem. Toxicol. 49, 5: 1096-101.

Ranneh, Y, Ali, F, Zarei, M, Akim, A, Hamid, HA, et al, 2018: Malaysian stingless bee and
Tualang honeys: A comparative characterization of total antioxidant capacity and phenolic profile using liquid chromatography-mass spectrometry. LWT-Food Sci. Tech. 89:1-9.

Rasul, A, Millimouno, FM, Eltayb, WA, Ali, M, Li, J, et al, 2013: Pinocembrin: A novel natural compound with versatile pharmacological and biological activities. Bio. Med. Res. Int. 13:1-9.

Rezanejad, F, 2009: Air pollution effects on structure, proteins and flavonoids in pollen grains of Thuja orientalis L. (Cupressaceae). Grana 48: 205-13.

Robles, C, Greff, S, Pasqualini, V, Garzino, S, Bousquet, A, et al, 2003: Phenols and flavonoids in Aleppo Pine needles as bio-indicators of air pollution. J. Environ. Quality 32:2265-71.

Šarić, A, Balog, T, Sobočanec, S, Kušić, B, Šverko, V, et al, 2009: Antioxidant effects of flavonoid from Croatian Cystus incanus L. rich bee pollen. Food Chem. Toxicol. 47:547-54.

Siage, J, 1976: Considerations on the treatment of psoriasis. Ann. Derm. Syph. 103:610-6.

Sokhansanj, S, Jayas, DS, 1995: Drying of FoOd-Stuffs. In: Handbook of Industrial Drying. By Shahab Sokhansanj. 